\title{
Status of positron beams for dark photons experiments
}

\author{
Paolo Valente ${ }^{1, \star}$ \\ ${ }^{1}$ INFN Sezione di Roma, P.le Aldo Moro, 2, I-00185 Rome, Italy
}

\begin{abstract}
High energy positron beams are an important tool for fixed-target experiments searching for new particles produced in the annihilation on atomic electrons of a target. The status of existing or planned infrastructures is reviewed.
\end{abstract}

\section{Introduction}

The idea that the new dark particles are not directly connected with the Standard Model (SM) gauge fields, but only through mediators, linking a new, "secluded" sector to the ordinary one, has triggered a number of experiments, mainly devoted to search for a vector boson (or dark photon, $A^{\prime}$ ), kinetically mixed with the ordinary photon (with coupling $\alpha^{\prime}$ related to the electromagnetic one through the parameter $\left.\varepsilon^{2}=\alpha^{\prime} / \alpha\right)$.

Practically all the experimental results are based on the search for the decay into a SM fermion anti-fermion pair of the dark photon, being irradiated in the interaction of an electron beam with the nuclei of a target (in fixed-target experiments), or coming from the decay of a meson (in colliders).

In the so-called "visible" decays of the dark photon, detecting the decay of the $A^{\prime}$-boson to a SM fermion pair $\left(e^{+} e^{-}\right.$if $\left.m_{A^{\prime}}<2 m_{\mu}\right)$ requires a significant branching of $A^{\prime}$ decay to the lepton pair. This is indeed the only available channel if the dark photon is the lightest particle in the new sector. A complication of this method is the high level of electromagnetic background in the $e^{+} e^{-}$mass spectrum. In addition, a large number or results have been obtained both in fixed-target experiments, using $A^{\prime}$-strahlung production from high-intensity/high-energy electron beams, and in collider experiments, using meson decays to $\gamma A^{\prime}$.

In the hypothesis that $m_{A^{\prime}}<2 m_{\chi}$, being $\chi$ the lightest fermion in the dark sector, the most interesting range of the parameters space is already excluded [1]; in particular the mass range between 1 and $100 \mathrm{MeV} / c^{2}$ and the coupling down to $\varepsilon^{2} \sim 10^{-6}$ that could explain the discrepancy between theory and observation for the muon anomalous magnetic moment [2]. However, removing any modeldependent hyphotesis on the $A^{\prime}$ decay modes and mass, the parameter space is much less constrained.

Following the proposal by B. Wojtsekhowski [3], an alternative approach is to look for the "invisible" decays of the $A^{\prime}$, i.e. without requiring SM fermions in the final state, and exploiting the kinematics of the annihilation of a positron beam of well-known momentum onto the electrons of a target. Measuring the momentum of the ordinary photon in events $e^{+} e^{-} \rightarrow \gamma A^{\prime}$ would indeed allow the observation of a peak in the missing mass spectrum for $m_{\mathrm{m} i s s}^{2}=m_{A^{\prime}}^{2} \neq 0$, independently from the decay mode and life-time of the dark photon.

\footnotetext{
^e-mail: paolo.valente@roma1.infn.it
} 
In this approach, a high energy positron beam, with well defined quadri-momentum impinges on a thin (i.e. not fully absorbing the incoming beam), low- $Z$ target, in a well defined position and with the best possible reproducibility. Of course the sensitivity scales with the statistics, i.e. with the number of interactions of the incoming positrons with the target electrons.

The intensity of the beam is ultimately dictated by the pile-up probability in the downstream detector detecting the ordinary photon in the final state. Among the various background sources, mainly Bremsstrahlung on the target nuclei, $e^{+}(N) \rightarrow e^{+}(N) \gamma$, and two (three) photons process, $e^{+} e^{-} \rightarrow \gamma \gamma(\gamma)$ are the dominant ones.

The original idea proposed in Ref. [3] is to use the positron beam circulating in a collider ring (like the Novosibirsk VEPP-3 machine), striking it onto an internal gaseous Hidrogen target. This requires to have a internal target facility and the possibility of clearing the acceptance of the emitted ordinary photon in order to place a calorimeter at a suitable distance for measuring the angle and the energy with the required resolution.

A more flexible approach would be to use an extracted positron beam, in order to optimize both the experimental setup for the missing mass spectrum measurement, and the target, as proposed by the PADME [4, 5] and MMAPS [7] collaborations. It is important to note that the ideal time structure of the beam for the proposed annihilation experiments is to have a continuous positron beam, or at least a long beam pulse, in order to dilute the highest possible number of positron interactions, while keeping under control the probability of pile-up in the photon detector, taking into account both the $A^{\prime} \gamma$ signal and the other photon sources (Bremsstrahlung and $2 / 3 \gamma$ backgrounds).

The availability of a high-energy extracted beam would of course allow performing any other kind of positron fixed-target experiment, like searches of "visible" dark photon decays from $A^{\prime}$-strahlung, dump experiments, or other light dark matter searches, opening also the possibility (with respect to the circulating beam) of further manipulation (collimation, focussing, etc.). In the following some of the available or planned accelerator facilities with the possibility of having an extracted, high-energy ( $>$ few tens of $\mathrm{MeV}$ ) positron beam are briefly described.

\section{Laboratori Nazionali di Frascati positron beam}

\subsection{Beam-Test Facility (BTF)}

The Beam-Test Facility (BTF) is part of the LNF accelerator complex in operation since 2004 [8]. It makes use of the LINAC, used as injector of the Frascati $e^{+} e^{-}$high-luminosity collider DAФNE, running at the $\Phi$ meson resonance (center of mass energy of $1020 \mathrm{MeV}$ ).

The DAФNE linear accelerator [9] is a constant gradient, travelling wave, $2 / 3 \pi$, S-band (2856 $\mathrm{MHz}$ ) LINAC, composed by 15 SLAC-type, $3 \mathrm{~m}$ long, accelerating structures, fed by four radiofrequency power stations, each with a modulator supplying a $45 \mathrm{MWp}$ klystron (Thales TH-2128C) at the maximum repetition rate of $50 \mathrm{~Hz}$.

The electron source consists of a gridded triode gun with replaceable cathode. The LINAC has been designed on the model of the two-mile linear accelerator in SLAC. In order to get higher accelerating fields, each klystron is followed by a SLED (SLAC Energy Doubling) system using two coupled cavities, in order to compress the $4.5 \mu$ s long RF pulse.

The time duration of each LINAC pulse is dictated by the characteristics of the pulsing system, providing a rectangular waveform to the gun for the extraction of electrons emitted by the filament, kept at a fixed bias voltage. In the standard configuration [10], it is capable of delivering pulses of adjustable total width in the range 1.5 to $40 \mathrm{~ns}$. After acceleration, of course the beam will have a micro-bunched structure at the RF frequency of $2856 \mathrm{MHz}$. For the injection into the DAФNE rings, the optimal pulse duration is $10 \mathrm{~ns}$, while the typical beam charge is of $0.5 / 1 \mathrm{nC}$ for electron/positron 
pulses. Un upgraded pulsing system has been recently commissioned, allowing to deliver pulses of increasing length, up to $5 \mu \mathrm{s}$, so that - after having optimized the RF power and phases and the magnetic focussing in the LINAC - electron or positron beams could get accelerated close to the maximum energy, with a width of several hundreds of ns.

After the gun, electrons are bunched and brought to $v=c$ by pre-buncher and buncher cavities, followed by the five $3 \mathrm{~m}$ long, standard SLAC sections, accelerating them up to $\sim 220 \mathrm{MeV}$. Positrons can then be produced by a two radiation lengths Tungsten-Rhenium converter, followed by solenoids for focussing and a magnetic chicane for electron separation and a high-field accelerating section (capture section); together with the remaining nine standard sections, positrons can be accelerated up to $\sim 550 \mathrm{MeV}$.

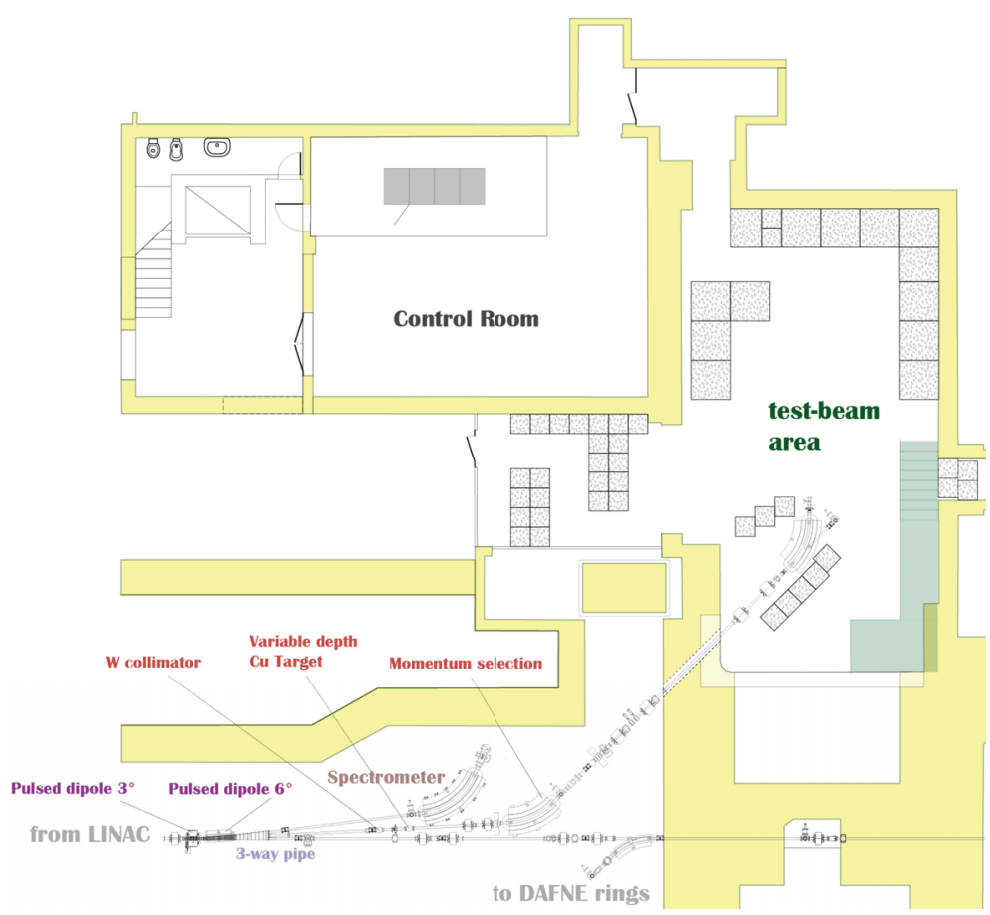

Figure 1. General layout of the Beam-Test Facility (BTF) of the Frascati DAФNE accelerator complex.

As shown in the scheme of Fig. 1, at the end of the LINAC the electron/positron beam can be diverted in three different lines using two pulsed magnets:

- At $0^{\circ}$, to the transfer-line to the damping ring (and from there, back to the pulsed magnet driving it to the transfer-line towards the DAФNE main rings);

- at $3^{\circ}$, to the BTF beam-line;

- at $6^{\circ}$, to the spectrometer line: a $60^{\circ}$ dipole plus a segmented (metallic strip) detector allows measuring the beam energy with a $<0.5 \%$ accuracy.

The DAФNE timing system can realize different sequences in which a portion of the 50 pulses available in one second are injected to the damping ring, while the remaining ones are deflected on the BTF line. At least one pulse (two during the extraction from the damping ring and injection to the 
main rings, which is performed at $2 \mathrm{~Hz}$ ) is sent to the spectrometer line, in order to have a $1 \mathrm{~Hz}$ measurement of the LINAC beam energy. A dedicated sequence for the standalone operation of the BTF is available, sending all available 49 pulses to the test-beam, and is used when not injecting for the collider operation. The resulting time structure for the positron (or electron) beam extracted to the BTF line is shown in Fig. 2, yielding a duty-factor of $40 \mathrm{~ns} / 20 \mathrm{~ms}=2 \cdot 10^{-6}$.

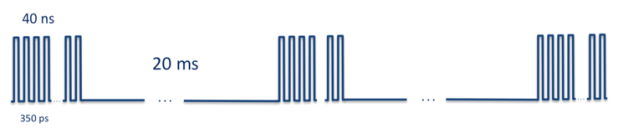

Figure 2. Time structure of the BTF extracted beam.

Since there is only one transfer line from the LINAC to the damping ring (also used for the reverse path from the damping ring to the main rings), it is necessary to reverse the polarity of all magnetic elements when switching from the injection of electrons to positrons, and viceversa. In this switching time the LINAC cannot deliver particles. This is a not negligible limitation of the duty-cycle of the BTF beam-line, when operating it together (in the so-called "parasitic mode") with the $e^{+} e^{-}$collider.

It is worth noticing that it is possible either to deliver to the BTF line the full LINAC beam, i.e. at high intensity and at the fixed energy of the LINAC (510 MeV during collider operation), or producing secondary particles intercepting the LINAC beam with a Copper target, and then selecting the charge and momentum of secondaries with a $43^{\circ}$ dipole magnet plus collimators.

Together the focussing system of the BTF line, this also results in a strong and tunable attenuation of the beam, so that electrons or positrons can be delivered in the full energy range down to the "single particle" regime, i.e. of a Poisson distribution with adjustable average number of particles. As shown in Fig. 1, two FODO quadrupole doublets and a final $45^{\circ}$ dipole, allow driving and focussing the beam in the BTF test-area, shielded by moveable concrete blocks. Taking into account the all different operation modes, the resulting sets of parameters for the BTF beam are shown in Tab. 1 .

\begin{tabular}{|c|c|c|c|c|}
\hline Parameter & \multicolumn{4}{|c|}{ Values } \\
\hline Maximum flux & \multicolumn{4}{|c|}{$3.125 \cdot 10^{10} \mathrm{~s}^{-1}$} \\
\hline Spot size (hor.) & \multicolumn{4}{|c|}{$0.7-55 \mathrm{~mm}$} \\
\hline Spot size (vert.) & \multicolumn{4}{|c|}{$0.7-25 \mathrm{~mm}$} \\
\hline Divergence & \multicolumn{4}{|c|}{$1-2 \mathrm{mrad}$} \\
\hline Parameter & \multicolumn{2}{|c|}{ Parasitic mode } & \multicolumn{2}{|c|}{ Dedicated mode } \\
\hline Pulse duration & \multicolumn{2}{|c|}{$10 \mathrm{~ns}$} & \multicolumn{2}{|c|}{$1.5-40 \mathrm{~ns}$} \\
\hline \multirow[t]{2}{*}{ Repetition rate } & \multicolumn{2}{|c|}{$\begin{array}{c}\text { Variable } \\
\text { (depending on DAФNE injection }\end{array}$} & \multicolumn{2}{|c|}{$\begin{array}{c}1-49 \mathrm{~s}^{-1} \\
(\text { selectable) }\end{array}$} \\
\hline & $\begin{array}{c}\text { With } \\
\text { target }\end{array}$ & $\begin{array}{l}\text { Without } \\
\text { target }\end{array}$ & $\begin{array}{l}\text { With } \\
\text { target }\end{array}$ & $\begin{array}{c}\text { Withtaout } \\
\text { target }\end{array}$ \\
\hline $\begin{array}{l}\text { Particle type } \\
\left(e^{+} \text {or } e^{-}\right)\end{array}$ & $\begin{array}{l}\text { selectable } \\
\text { by users }\end{array}$ & $\begin{array}{c}\text { alternating } \\
\text { according to } \\
\text { DAФNE status }\end{array}$ & $\begin{array}{l}\text { selectable } \\
\text { by users }\end{array}$ & $\begin{array}{l}\text { selectable } \\
\text { by users }\end{array}$ \\
\hline$e^{-}$Energy (MeV) & $25-500$ & 510 & $25-700$ & $250-730$ \\
\hline$e^{+}$Energy $(\mathrm{MeV})$ & $25-500$ & 510 & $25-500$ & $250-530$ \\
\hline Energy spread & $1 \%$ at $500 \mathrm{MeV}$ & $0.5 \%$ & $0.5 \%$ & $0.5 \%$ \\
\hline Intensity & $1-10^{5}$ & $10^{7}-1.5 \cdot 10^{10}$ & $1-10^{5}$ & $10^{3}-3 \cdot 10^{10}$ \\
\hline
\end{tabular}

Table 1. Beam parameters achievable in all possible operation modes at the end of the BTF line. 
The present BTF target is shaped so that three different thickness values can be selected, corresponding to $1.7,2$ or 2.3 radiation lenghts. Positrons produced by high-energy primary electrons striking such a target exhibit a typical energy-angle anti-correlation: higher energy positrons will be produced mostly in the forward direction, while lower energy ones will be spread over a much wider angle distribution. Considering the effect of the collimation and the magnetic focussing, it is possible to optimize the yield of high energy positrons (close to $E_{0}$, the incoming primary electron energy) as a function of the thickness of the target, as shown in Fig. 3. This suggests that a thinner Copper target (of the order of one radiation length) with respect to the present one, can improve the number of produced positrons starting from primary electrons accelerated - exploiting the full length of the LINAC - to the maximum energy of about $750 \mathrm{MeV}$. Recently, an upgrade of the LINAC energy up to $1 \mathrm{GeV}$ has been also proposed [11].

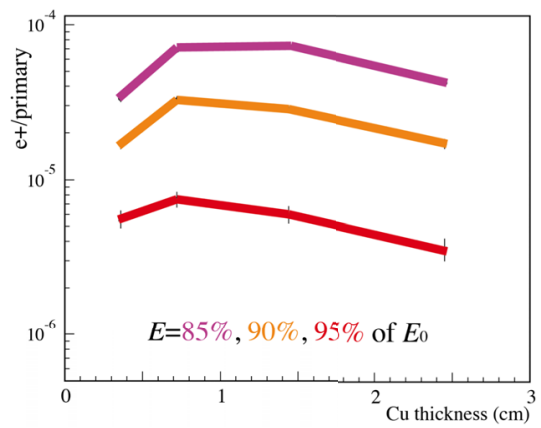

Figure 3. Positron yield as a function of the BTF target depth (Copper).

Studies are currently under way for extending the accelerated positron beam duration above $40 \mathrm{~ns}$ (up to $200 \mathrm{~ns}$, resulting in a duty-factor of $\approx 10^{-5}$.), in order to increase the luminosity of fixed-target annihilation experiments while keeping the pile-up probability constant. The length of electron (and positron) pulses from the LINAC is limited by the peculiar, peaked shape of the accelerating voltage due to the compression of the RF power by the SLED system, so that a dedicated development for tuning the RF power, phase and delays in all the sections of the LINAC has to be performed, on the model of what achieved in Ref. [12].

\subsection{Possible extracted beam from DA $\Phi$ NE}

An alternative to the extraction of the LINAC beam in the BTF line would be to perform a slow extraction from the DAФNE collider ring, of $510 \mathrm{MeV}$ positrons. The $e^{+} e^{-}$collider is composed by two separated rings (as schematically shown in Fig. 4), one for the electron and one for the positron beam, intersecting in one interaction region (IP1) and vertically separated on the opposite side of the machine. The RF frequency for the $97.7 \mathrm{~m}$ long rings is of $368.26 \mathrm{MHz}$, corresponding to up to 120 bunches, spaced by $2.7 \mathrm{~ns}$. The positron stored current can exceed $1 \mathrm{~A}$, with $\beta_{x} \simeq 1.6 \mathrm{~m}, \beta_{y} \simeq 17 \mathrm{~mm}$, and emittance $0.34 \cdot 10^{-6}$.

The slow extraction could be realized by using the third order resonance, excited by means of a sextupole and tuned by quadrupoles, together with RF knockout and septum magnet. Detailed studies are needed in order to design the extraction layout and thus estimate the parameters of the extracted beam; however good emittance and beam energy spread are expected, while the extraction efficiency and spill length has to be carefully calculated. 


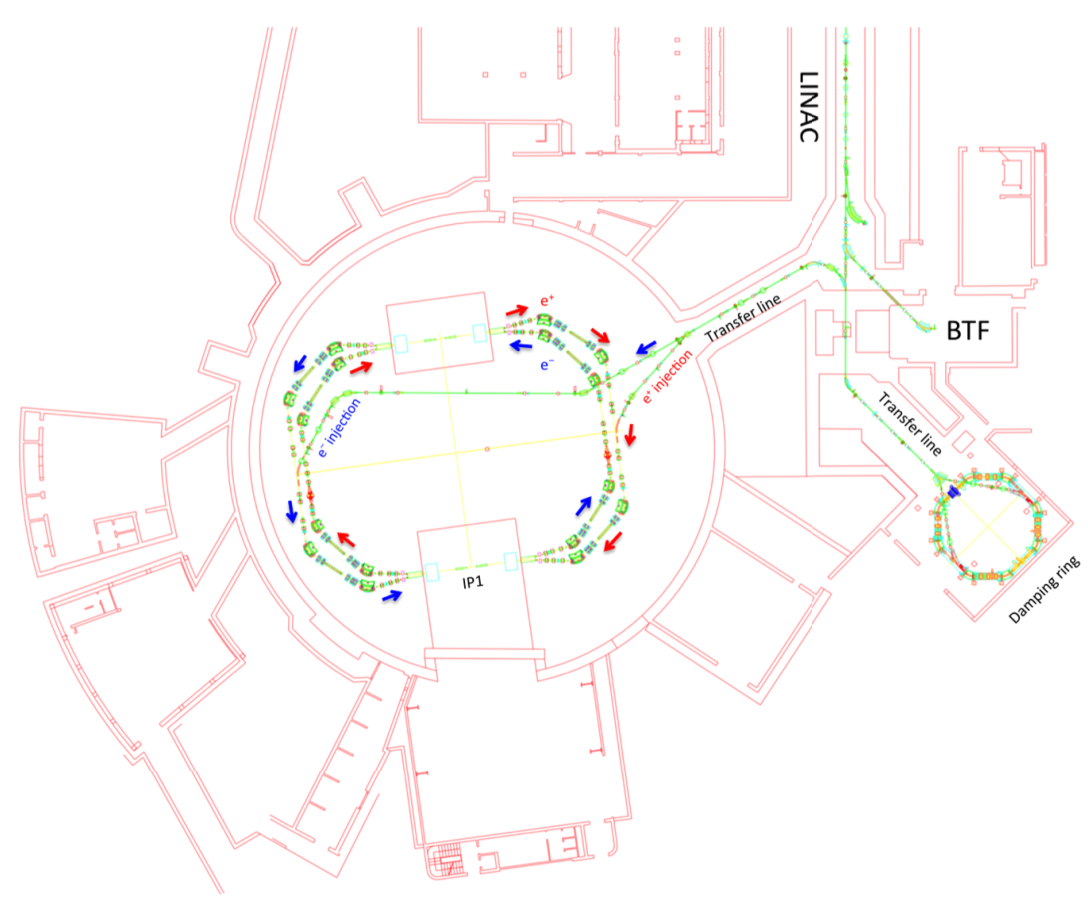

Figure 4. DA $\Phi N E$ accelerator complex in Frascati, showing the two positron and electron intersecting rings.

\section{Other facilities}

At SLAC, FACET-II is a new test facility to develop advanced acceleration and coherent radiation techniques with high-energy electron and positron beams, representing a major upgrade over current FACET capabilities. The FACET-II project has two stages: first a new photoinjector and two bunch compressors in the LINAC will restore operation with electrons; then a new positron damping ring with injection and extraction lines will restore operation with positrons. Positrons are accelerated to $10 \mathrm{GeV}$, compressed and focused to a transverse size of $<20 \mu \mathrm{m}$ at the interaction point and dumped. Repetition rate of the LINAC will be $30 \mathrm{~Hz}$, and the planned charge is $1 \mathrm{nC}$ for positrons produced by $4 \mathrm{nC}$ of electrons, using the existing target and capture section [6].

\subsection{Proposed extracted beam at Cornell}

The Cornell Electron Storage Ring (CESR) has been operational as $e^{+} e^{-}$collider until the late 2000's, and is now used as a synchrotron radiation source (CHESS). Electrons produced by a thermo-ionic gun are accelerated and then driven onto a Tungsten target for producing positrons, then accelerated by the LINAC to $150 \mathrm{MeV}$. Up to 16 positron bunches from the LINAC are loaded into the Cornell Synchrotron and accelerated up to $5.3 \mathrm{GeV}$. From there positrons are then injected into the external storage ring, which includes the magnets for the production of synchrotron radiation. The average circulating current is $\approx 10 \mathrm{nA}$.

By using the slow extraction at $60 \mathrm{~Hz}$, quasi-continuous spills of $\approx 2 \mathrm{~ms}$ length, $\approx 2.5 \mathrm{~mA}$ current, of $5.3 \mathrm{GeV}$ positrons ( $6 \mathrm{GeV}$ after the planned upgrade of the complex, after the removal of the straight section for high-energy experiments like CLEO and CLEO-c) can be driven to a beam-line 


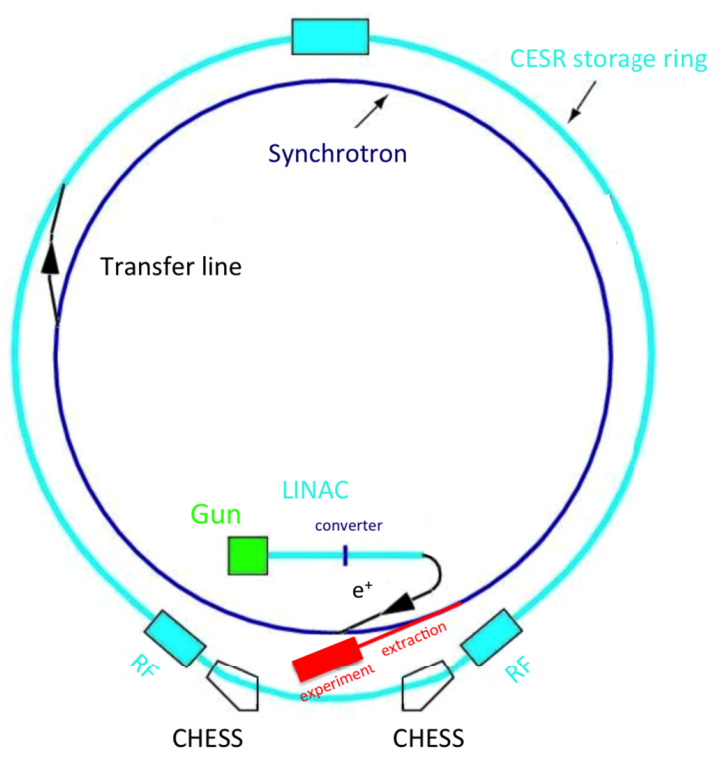

Figure 5. Proposed Cornell extracted beam for dark photon experiments.

for fixed-target experiments. Pulsed quadrupoles will shift the tune towards a third integer resonance, then pulsed sextupole will reduce the stable phase space pushing positrons away from the stable orbit (spiralizing outwards), where a septum can kick them out. The layout of the slowly extracted positron line from the synchrotron and the possible location of fixed-target experiments in between the two rings, is shown in Fig. 5, together with the production and acceleration chain (LINAC followed by synchrotron).

The beam time structure is made up by $\approx 12,000$ bunches, spaced by $168 \mathrm{~ns}$, repeated every 16.67 $\mathrm{ms}\left(60 \mathrm{~Hz}\right.$ repetition rate), resulting in a duty-factor of $\approx 10^{-1}$.

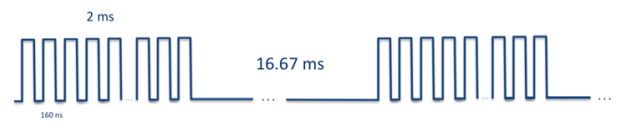

Figure 6. Time structure of the proposed Cornell extracted beam.

\subsection{VEPP-3}

Positrons are produced and accelerated up to $\approx 500 \mathrm{MeV}$ in the VEPP-3 ring, part of the Budker Institute for Nuclear Physics (BINP) in Novosibirsk. In particular, 6 bunches, 1 ns long and spaced by $41.5 \mathrm{~ns}$, of $\approx 2 \cdot 10^{10}$ positrons are injected and accumulated into the VEPP-3 storage ring. Positron annihilations can be realized by installing an internal gas target. The project is to use one of the straight sections of the machine for building a dedicated by-pass line (using three dipoles and six quadrupoles), as shown in the schematic layout of Fig. 7 and further discussed in Ref. [14]. 


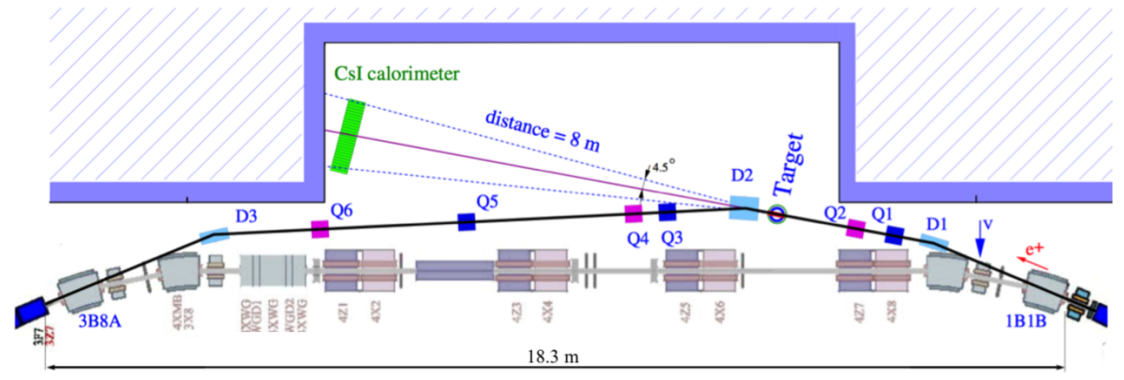

Figure 7. Proposed by-pass line at VEPP-3 in Novosibirsk.

\section{Acknowledgments}

This work is supported by the project PGR-226 of the Italian Ministry of Foreign Affairs and International Cooperation (MAECI), CUP I86D16000060005.

\section{References}

[1] J. Alexander et al., "Dark Sectors 2016 Workshop: Community Report,” arXiv:1608.08632 [hep$\mathrm{ph}]$.

[2] M. Pospelov, Phys. Rev. D 80 (2009) 095002.

[3] B. Wojtsekhowski, D. Nikolenko and I. Rachek, "Searching for a new force at VEPP-3," arXiv:1207.5089 [hep-ex].

[4] M. Raggi and V. Kozhuharov, Adv. High Energy Phys. 2014 (2014) 959802.

[5] M. Raggi, V. Kozhuharov and P. Valente, EPJ Web Conf. 96 (2015) 01025.

[6] V. Yakimenko et al., doi:10.18429/JACoW-IPAC2016-TUOBB02

[7] J. Alexander et al., "Cornell Dark Photon Search", APS April Meeting (2015), 2015APS..APRU15006A.

[8] A. Ghigo, G. Mazzitelli, F. Sannibale, P. Valente and G. Vignola, Nucl. Instrum. Meth. A 515 (2003) 524.

[9] B. Buomomo and L. Foggetta, IPAC 2015, Richmond (USA), TUPWA057 (2015).

[10] B. Buomomo, L. Foggetta and G. Piermarini, IPAC 2015, Richmond (USA), TUPWA056 (2015).

[11] P. Valente et al., "Linear Accelerator Test Facility at LNF: Conceptual Design Report," arXiv:1603.05651 [physics.acc-ph].

[12] F. J. Decker, Z. D. Farkas and M. G. Minty, SLAC-PUB-8111.

[13] S. Guiducci, DAФNE Technical Note G-73 (2017).

[14] I. Rachek, D. Nikokenko and B. Wojtsekhowski, these Proceedings. 